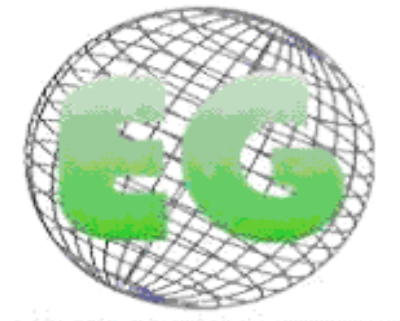

ISSN 1695-6141 N'25.
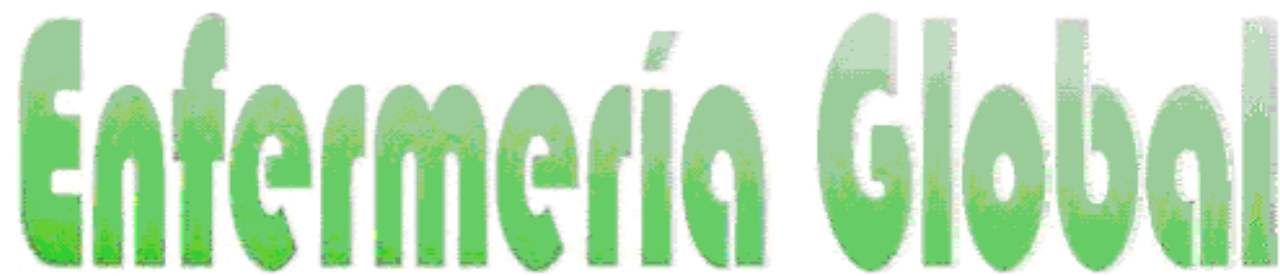

Revista electrónica trimestral de Enfermería

Enero 2012

www.um.es/egloball

\title{
REVISIONES
}

\section{Esquizofrenia y dependencia del tabaco: una revisión integradora}

Esquizofrenia e dependência de tabaco: uma revisão integrativa

\section{*Marques de Oliveira, R., " ${ }^{* *}$ Ferreira Furegato, AR.}

*Mestranda do Programa de Enfermagem Psiquiátrica da Escola de Enfermagem de Ribeirão Preto (EERPUSP). Bolsista do Conselho Nacional de Desenvolvimento Científico e Tecnológico (CNPQ). E-mail: renatamarques@ymail.com. ** Doutora. Professora titular do Departamento de Enfermagem Psiquiátrica e Ciências Humanas da Escola de Enfermagem de Ribeirão Preto (EERP-USP). Brasil.

Palabras clave: esquizofrenia; tabaquismo; enfermería psiquiátrica.

Palavras-chave: esquizofrenia; tabagismo; enfermagem psiquiátrica

Keywords: schizophrenia; smoking; psychiatric nursing

\section{RESUMEN}

El tabaquismo es una enfermedad crónica con perjuicio a la salud del cuerpo, mental, social y económica del usuario. Este estudio tuvo como objetivo identificar los conocimientos en la literatura científica producida sobre la dependencia del tabaco en la esquizofrenia y su utilización por la enfermería. Fue realizada una revisión integradora de la literatura científica, desde mayo 2001 hasta mayo 2011, desde las siguientes preguntas: ¿Existe relación entre la dependencia del tabaco y la esquizofrenia? ¿Esta temática ha sido abordada por la enfermería? Los artículos fueron seleccionados en el MEDLINE y en el Portal de Periódicos CAPES, siguiendo las palabras claves: tabaquismo, esquizofrenia y enfermería. De los 346 artículos encontrados, teniendo en cuenta los criterios, la muestra se quedó con 52 artículos, analizados y discutidos. Se constató que la relación entre la esquizofrenia y el tabaquismo es incuestionable según la literatura científica y preocupante, pues a pesar de los aparentes beneficios (mejora de los síntomas negativos, disminución de los efectos secundarios de los medicamentos, los sentimientos de placer) interfiere en el tratamiento de las drogas y perjuicio en la presentación del trastorno. De los 52 artículos analizados, solamente tres han hecho mención al profesional enfermero, lo que muestra una falta de posición de la enfermería en este tema. Se concluye que la asociación del tabaquismo y la esquizofrenia es preocupante y que el enfermero debe incluir el abordaje al fumador como parte del cuidado de la enfermería, ofreciendo a los sujetos pacientes psiquiátricos la oportunidad de refllexionar sobre el tabaquismo en su vida y su real disposición para recibir ayuda para dejar el hábito de fumar. 


\section{RESUMO}

O tabagismo é uma doença crônica com prejuízos à saúde física, mental e à vida social e econômica do usuário. Este estudo teve por objetivo, identificar na literatura científica o conhecimento produzido sobre a dependência de tabaco na esquizofrenia e sua utilização pela enfermagem. Foi realizada uma revisão integrativa da literatura científica, de maio de 2001 a maio de 2011, a partir das seguintes questões norteadoras: Existe relação entre dependência de tabaco e esquizofrenia? Esta temática tem sido abordada pela enfermagem? Os artigos foram selecionados no MEDLINE e no Portal de Periódicos da CAPES, a partir dos descritores: tabagismo, esquizofrenia e enfermagem. Dos 346 artigos encontrados, atendendo aos critérios, a amostra ficou constituída por 52 artigos, analisados e discutidos. Constatou-se que a relação entre esquizofrenia e tabagismo é inquestionável segundo a literatura científica e preocupante, pois apesar dos aparentes benefícios (melhora dos sintomas negativos, diminuição dos efeitos colaterais das medicações, sensação de prazer) há interferência na terapêutica medicamentosa e prejuízo na apresentação do transtorno. Dos 52 artigos analisados, apenas três fizeram menção ao profissional enfermeiro, evidenciando a falta de posicionamento da enfermagem diante desta temática. Conclui-se que a associação entre tabagismo e esquizofrenia é preocupante e que a enfermagem deve incluir a abordagem ao tabagista como parte do cuidado de enfermagem, oferecendo aos pacientes psiquiátricos a oportunidade de refletir sobre o tabagismo em sua vida e sobre sua real disposição para receber ajuda para abandonar o hábito.

\section{ABSTRACT}

Smoking is a chronic disease with damages to physical and mental health and to the social and economic life of the user. This study aimed to identify in the scientific literature the knowledge produced about tobacco dependence in schizophrenia and its utilization by nursing. An integrative review of the scientific literature was conducted, from May 2001 to May 2011, based on the following guiding questions: Is there a relationship between tobacco dependence and schizophrenia? Has this issue been approached by nursing? The papers were selected in MEDLINE and CAPES Periodicals Portal, from the descriptors: smoking, schizophrenia and nursing. Of the 346 papers found, attending to the inclusion criteria, the sample comprised52 papers, which were analyzed and discussed. It was found that the relationship between schizophrenia and smoking is unquestionable according to the scientific literature, and worrying because despite the apparent benefits (improvement of the negative symptoms, decrease of the adverse effects of the medicines, feeling of pleasure) there is interference in the drug therapy and impairment of disorder presentation. Of the 52 papers analyzed, only three mentioned the professional nurse, so evidencing the lack of positioning of nursing at the head of this issue. It was concluded that the association between smoking and schizophrenia is worrying and that nursing should include the approach to smoker as part of nursing care, offering psychiatric patients the opportunity to reflect on smoking in their life and on their willingness to receive help to quit the habit.

\section{INTRODUCCIÓN}

El tabaquismo es una enfermedad crónica, reconocida como un problema de salud pública y también la principal causa de muerte prevenible. Es una pandemia, responsable de cinco millones de muertes al año con perjuicios a la salud del cuerpo, mental, a la vida social y económica del usuario ${ }^{(1,2)}$.

Las organizaciones políticas han reconocido la gravedad del tabaquismo en la población mundial, proponiendo estrategias para intentar reducir este problema, logrando reducir la prevalencia del tabaquismo en las estadísticas nacionales y mundiales. Sin embargo, se percibe que el mismo resultado no ha sido alcanzado entre la población con trastorno mental; los esquizofrénicos presentan una prevalencia del tabaquismo dos o tres veces superior a la población general con mayor grado de dependencia nicotínica y mayor número de recaídas durante los períodos de abstinencia ${ }^{(1,3-6)}$.

Los estudiosos del tema reconocen que la enfermería es una profesión con condiciones para abordar este tema junto a los pacientes esquizofrénicos debido a la atención directa, la relación terapéutica y el abordaje comprensivo presente en su práctica diaria. No obstante, a pesar de esta posición privilegiada, la ausencia de conocimiento científico, así como la falta 
de referencias en las prácticas educativas sobre promoción de la salud se han mostrado como una seria barrera que limita sus acciones ${ }^{(7-13)}$.

Ante esa perspectiva, una de las posibilidades para los enfermeros que prestan asistencia a los esquizofrénicos fumadores consiste en la Práctica Basada en Evidencias (PBE) que busca el conocimiento científico como base de su práctica diaria.

En la búsqueda del conocimiento científico y en la toma de decisiones juiciosas, el enfermero puede encontrar las mejores alternativas para mejorar la calidad del cuidado, respetando las necesidades y preferencias del paciente de acuerdo con lo que espera la sociedad $^{(14-16)}$.

Así este estudio tuvo como objetivo identificar los conocimientos en la literatura científica producida sobre la dependencia del tabaco en la esquizofrenia y su utilización en la práctica diaria de la enfermería.

\section{METODOLOGÍA}

Para lograr los objetivos de este estudio, la búsqueda del conocimiento producido sobre esta temática estudiada, se realizó una revisión integradora de la literatura científica.

La revisión integradora es un método utilizado desde la década de 1980, de acuerdo con los presupuestos de la Práctica Basada en Evidencias, pues tiene por objetivo sintetizar el conocimiento científico sobre un determinado tema, basado en el análisis de estudios ya publicados (empíricos y teóricos), lo que posibilita al profesional, insertado en la práctica de los servicios de salud, una visión profundizada y vasta sobre el tema de interés, lo que favorece el proceso de toma de decisión y la mejoa de los cuidados ${ }^{(17-19)}$.

La revisión integradora es un método de investigación adecuado para el conocimiento de la producción en enfermería. Este procedimiento posibilita la discusión de experiencias ya realizadas, la identificación de las lagunas existentes en el conocimiento producido y la necesidad de realización de nuevos estudios. Permite la disponibilidad del conocimiento para los enfermeros que encuentran dificultades en el acceso a esos estudios, debido a la restricción de tiempo y de preparación para la realización de este tipo de análisis ${ }^{(18,19)}$.

Esta revisión integradora siguió las etapas recomendadas por los estudiosos del tema ${ }^{(18,19)}$ : 1) la formulación de la pregunta orientadora; 2) establecimiento de los criterios de inclusión y exclusión de los estudios (proceso de muestreo); 3) definición de las informaciones que se extraen de los estudios seleccionados; 4) evalaución de los estudios incluidos; 5) interpretación de los resultados y 6) presentación de la revisión.

Las preguntas orientadoras para este estudio fueron: ¿Existe relación entre la dependencia del tabaco y la esquizofrenia? ¿Esta temática ha sido abordada por la enfermería?

Los artículos fueron seleccionados en el MEDLINE (Medical Literature Analysis and Retrieval System online) y en el Portal de Periódicos CAPES, siguiendo las palabras claves: tabaquismo, esquizofrenia y enfermería.

Criterios de inclusión: todos los artículos publicados en su totalidad en el periodo entre mayo de 2001 a mayo de 2011, en los idiomas portugués, inglés y español.

Criterios de exclusión: 1) aspectos genéticos, inmunológicos, farmacológicos, neuroestructurales y neurotransmisión del tabaquismo en la esquizofrenia; 2) tabaquismo en 
la esquizofrenia como factor de riesgo para perjuicios físicos y el aumento de la mortalidad; 3) discusión sobre el cese del tabaquismo en los esquizofrénicos; 4) relación entre el tabaquismo y esquizofrenia como aspecto secundario a otras variables (el uso de cafeína, otras sustancias psicoactivas, personalidad) y 5) restricciones al tabaquismo en los servicios de internación psiquiátrica.

Fueron encontrados en el MEDLINE, a partir de la combinación de las palabras claves, 346 artículos. Después de la lectura del título y el resumen de cada uno de los artículos, 294 (85\%) fueron excluidos: $55,4 \%$ de esos abordaron aspectos genéticos, inmunológicos, farmacológicos, neuroestructurales y de neurotransmisión; 13,6\% trataban el tabaquismo como factor de riesgo para perjuicios físicos y el aumento da mortalidad; $7,1 \%$ discuten sobre el cese del tabaquismo en esta población; 18,7\% discutían la relación entre el tabaquismo y la esquizofrenia como aspecto secundario a otras variables (el uso de cafeína, otras sustancias psicoactivas, personalidad); $0,3 \%$ abordaron las restricciones al tabaquismo en los servicios de internación psiquiátrica; $4,8 \%$ no estaban disponibles en su totalidad en las dos bases de datos.

La muestra final fue de 52 artículos (15\%), siendo que 50 (96\%) no estaban disponibles en su totalidad en el MEDLINE, por lo tanto, fueron encontrados en el Portal de Periódicos de CAPES.

Para los artículos seleccionados en la muestra final $(n=52)$ fue utilizado un cuadro, basado en el instrumento desarrollado por Ursi y Galvão ${ }^{(20)}$ que proponen la siguiente organización: nombre del artículo, autores, intervención estudiada, resultados, recomendaciones/ conclusiones. Para ese estudio fueron realizadas modificaciones en la nomenclatura (el nombre del artículo fue nombrado título, la intervención estudiada fue nombrada metodología) y en el diseño de la tabla (los resultados y conclusiones fueron organizados en solo una columna y el nombre de los autores no se incluyó, siendo mencionado solo el título del artículo, el año y la referencia, de acuerdo con las normas del periódico).

El análisis de los 52 artículos ocurrió de modo descriptivo, buscando identificar en la literatura científica el conocimiento producido sobre la dependencia del tabaco en la esquizofrenia y cómo este tema ha sido abordado en la práctica diaria de la enfermería. Los artículos también fueron analizados en relación a la clasificación jerárquica de las evidencias, según la Agencia de Investigación y Calidad en Salud de los Estados Unidos ${ }^{(21)}$.

\section{RESULTADOS}

De los 52 artículos analizados, 44 (84,6\%) eran de diseño empírico (investigaciones de campo) y ocho $(15,4 \%)$ estaban constituidos por revisiones de literatura y discusiones teóricas. Los estudios con diseño empírico $(n=44)$ fueron conducidos en 18 países: 34\% en los Estados Unidos, 13,6\% en China, 9\% en Finlandia, 4,5\% en España, 4,5\% en Suiza, $4,5 \%$ en Turquía y 11 (25\%) en Israel, Canadá, Australia, Alemania, Egipto, Suecia, Colombia, Hungría, Grecia, Francia e India. No se identificó ningún estudio de diseño empírico conducido en Brasil.

De los artículos analizados, 50 (96\%) estaban redactados en inglés y dos en portugués (4\%). Los principales periódicos que publicaron estos estudios fueron Schizophrenia Research (25\%) y Psychiatry Research (13,5\%), con factor de impacto 4.374 y 2.803 , respectivamente. 
De los 44 artículos con diseño empírico, 24 (54,5\%) fueron conducidos en la comunidad, 16 $(36,4 \%)$ en servicios de internación psiquiátrica y cuatro $(9,1 \%)$ en la comunidad y servicios de internación.

La metodología cuantitativa fue la más empleada (82,7\%), teniendo $15,4 \%$ de revisiones de literatura/discusiones teóricas y 1,9\% metodología cualitativa. Los estudios fueron clasificados según el nivel de evidencia 3 (54\%), nivel de evidencia 4 (25\%) y nivel de evidencia 5 (15\%). Fue identificado un meta-análisis (nivel de evidencia 1), un estudio con diseño experimental (nivel de evidencia 2) y una opinión de un especialista (nivel de evidencia 6).

El Examen de Dependencia Nicotínica de Fagerstrom fue utilizado en 36,4\% de los estudios con diseño empírico.

Sin embargo, el descriptor de enfermería ha sido utilizado en la búsqueda de artículos en las bases de datos, encontrándose solo tres artículos $(5,7 \%)$ que mencionaron al profesional enfermero, todos publicados en lengua inglesa y clasificados con el nivel de evidencia 5.

Diferentes definiciones fueron utilizadas en los estudios en relación al término fumador. Para $12 \%$, fumador es el individuo que fumó todos los días en el último mes; para 4\%, fumador es el individuo que fumó más de 100 cigarrillos a lo largo de su vida y fumó en el último mes; para $4 \%$, fumador es el individuo que está fumando actualmente; para $2 \%$, fumador es el individuo que fuma semanalmente o con más frecuencia. Mientras, $79 \%$ no explicitaron la definición utilizada.

Los dos cuadros abajo presentan las principales informaciones obtenidas de los 44 artículos con diseño empírico y de los ocho artículos teóricos y de revisión.

Cuadro I - Principales informaciones obtenidas de los estudios con diseño empírico

\begin{tabular}{|c|c|c|}
\hline Título (año) & Metodología & Resultados/Conclusión \\
\hline $\begin{array}{l}\text { Smoking rates } \\
\text { among } \\
\text { schizophrenia } \\
\text { patients in Japan } \\
(2011)^{(22)}\end{array}$ & $\begin{array}{l}172 \text { esquizofrénicos, internados } \\
\text { en hospital psiquiátrico, y } 7496 \\
\text { controles de la población de } \\
\text { Japón fueron cuestionados } \\
\text { acerca del tabaquismo, } \\
\text { respondieron el examen de } \\
\text { Fagerstrom y tuvieron la } \\
\text { concentración de monóxido de } \\
\text { carbono medida en la } \\
\text { expiración. }\end{array}$ & $\begin{array}{l}40,7 \% \text { de los esquizofrénicos y } 24,2 \% \\
\text { de los controles eran tfumadores. } \\
\text { Entre los esquizofrénicos del sexo } \\
\text { masculino, el tabaquismo fue más } \\
\text { frecuente en la franja etaria de los } 50 \text { - } \\
59 \text { años y entre las mujeres en la } \\
\text { franja etaria de los } 40-49 \text { años. La } \\
\text { asociación entre tabaquismo y } \\
\text { esquizofrenia es consistente entre los } \\
\text { países, incluyendo Japón. }\end{array}$ \\
\hline $\begin{array}{l}\text { Gender-specific } \\
\text { profiles of } \\
\text { tobacco use } \\
\text { among non- } \\
\text { institutionalized } \\
\text { people with } \\
\text { serious mental } \\
\text { illness (2010) }\end{array}$ & $\begin{array}{l}729 \text { sujetos con trastornos } \\
\text { mentales severos, residentes en } \\
\text { la comunidad, fueron } \\
\text { cuestionados acerca del } \\
\text { tabaquismo y tuvieron la } \\
\text { dependencia nicotínica medida } \\
\text { por el examen de Fagerstrom. }\end{array}$ & $\begin{array}{l}46,8 \% \text { de los sujetos eran fumadores } \\
\text { con alto grado de dependencia } \\
\text { nicotínica. La prevalencia de } \\
\text { tabaquismo entre los esquizofrénicos } \\
\text { fue de } 51,8 \% \text {. La mayor parte de la } \\
\text { renta es invertida en la compra de } \\
\text { cigarrillos y fuman para soportar los } \\
\text { síntomas psiquiátricos. }\end{array}$ \\
\hline $\begin{array}{l}\text { Premorbid } \\
\text { tobacco smoking } \\
\text { is associated }\end{array}$ & $\begin{array}{l}230 \text { esquizofrénicos del sexo } \\
\text { masculino, internados en } \\
\text { hospitales psiquiátricos, fueron }\end{array}$ & $\begin{array}{l}42,2 \% \text { de los esquizofrénicos eran } \\
\text { fumadores y } 46,4 \% \text { tenían alto grado } \\
\text { de dependencia nicotínica. } 90,7 \%\end{array}$ \\
\hline
\end{tabular}




\begin{tabular}{|c|c|c|}
\hline $\begin{array}{l}\text { with later age at } \\
\text { onset in } \\
\text { schizophrenia } \\
(2010)\end{array}$ & $\begin{array}{lr}\text { investigados al } & \text { respecto del } \\
\text { tabaquismo, } & \text { características } \\
\text { clínicas y funciones cognitivas, a } \\
\text { través de procedimientos } \\
\text { estructurados. }\end{array}$ & $\begin{array}{l}\text { comenzaron el tabaquismo antes del } \\
\text { trastorno. La larga duración de la } \\
\text { enfermedad fue asociada al alto grado } \\
\text { de dependencia. Los fumadores } \\
\text { tuvieron mejor desempeño en los } \\
\text { exámenes cognitivos (mejora de los } \\
\text { pérdidas cognitivas de la } \\
\text { esquizofrenia). }\end{array}$ \\
\hline $\begin{array}{l}\text { Cigarette } \\
\text { smoking in } \\
\text { patients with } \\
\text { schizophrenia in } \\
\text { China: } \\
\text { prospective, } \\
\text { multicentre study } \\
(2010)(25)\end{array}$ & $\begin{array}{l}374 \text { esquizofrénicos } \\
\text { ambulatoriales de China fueron } \\
\text { investigados con relación a } \\
\text { datos sociodemográficos, } \\
\text { tabaquismo, uso de alcohol, } \\
\text { síntomas positivos y negativos. } \\
\text { Fueron acompañados por un } \\
\text { período de uno a dos años. }\end{array}$ & $\begin{array}{l}13,9 \% \text { de los esquizofrénicos eran } \\
\text { fumadores. Variables asociadas: sexo } \\
\text { masculino, desempleo, consumo de } \\
\text { alcohol, edad avanzada, larga } \\
\text { duración de la enfermedad, mayor } \\
\text { número de internaciones psiquiátricas, } \\
\text { inquietud y hostilidad, menos síntomas } \\
\text { negativos y positivos. El índice del } \\
\text { tabaquismo en los esquizofrénicos } \\
\text { chinos fue inferior a los valores } \\
\text { demostrados en la literatura } \\
\text { occidental. }\end{array}$ \\
\hline $\begin{array}{l}\text { Smoking in } \\
\text { schizophrenia: } \\
\text { diagnostic } \\
\text { specificity, } \\
\text { symptom } \\
\text { correlates and } \\
\text { illness severity } \\
(2010)(26)\end{array}$ & $\begin{array}{l}542 \text { psicóticos, de Nueva York, } \\
\text { fueron acompañados durante } 10 \\
\text { años después de la primera } \\
\text { internación. Participaron en } \\
\text { cinco entrevistas estructuradas. } \\
\text { Se investigó: tabaquismo, } \\
\text { sintomas positivos y negativos, y } \\
\text { gravedad de la enfermedad. }\end{array}$ & $\begin{array}{l}67,4 \% \text { de los esquizofrénicos, } 69,2 \% \\
\text { de los bipolares y } 73,1 \% \text { de los } \\
\text { depresivos eran fumadores. Los } \\
\text { fumadores presentaron mayor } \\
\text { gravedad de la enfermedad en } \\
\text { comparación a los no tfumadores. }\end{array}$ \\
\hline $\begin{array}{l}\text { Reasons for } \\
\text { smoking among } \\
\text { individuals with } \\
\text { schizophrenia } \\
(2010)(27)\end{array}$ & $\begin{array}{l}80 \text { sujetos con esquizofrenia o } \\
\text { trastornos esquizoafectivos } \\
\text { fueron investigados con relación } \\
\text { a las razones para fumar, } \\
\text { número de cigarrillos/día, edad } \\
\text { del inicio y contestaron el } \\
\text { Examen de Fagerstrom. Fueron } \\
\text { comparados con } 463 \text { fumadores } \\
\text { del grupo control sin ningún } \\
\text { diagnóstico psiquiátrico. }\end{array}$ & $\begin{array}{l}\text { Los esquizofrénicos/esquizoafectivos } \\
\text { presentaron mayor dependencia } \\
\text { nicotínica que los controles y se } \\
\text { mostraron más propensos a fumar en } \\
\text { búsqueda del efecto estimulador y de } \\
\text { la mejora del afecto negativo. El } \\
\text { tabaquismo para los } \\
\text { esquizofrénicos/esquizoafectivos es } \\
\text { una acción consciente (un escape, } \\
\text { medio de aliviarse del estrés), al } \\
\text { contrario de los controles que fuman } \\
\text { por automaticidad/ hábito. }\end{array}$ \\
\hline $\begin{array}{l}\text { Sex difference in } \\
\text { the prevalence of } \\
\text { smoking in } \\
\text { Chinese } \\
\text { schizophrenia } \\
(2010) \text { (28) }\end{array}$ & $\begin{array}{l}510 \text { esquizofrénicos internados y } \\
793 \text { controles fueron } \\
\text { investigados en los datos } \\
\text { sociodemográficos, tabaquismo, } \\
\text { condiciones } \\
\text { médicas/psicológicas } \\
\text { monóxido de y } \\
\text { expiratorio. }\end{array}$ & $\begin{array}{l}56 \% \text { de los esquizofrénicos y } 50 \% \text { de } \\
\text { los controles eran fumadores. } \\
\text { Esquizofrénicos del sexo masculino } \\
\text { presentaron mayor prevalencia del } \\
\text { tabaquismo }(81 \%) \text { de que los sujetos } \\
\text { del sexo masculino del equipo control } \\
(66 \%) \text {. }\end{array}$ \\
\hline $\begin{array}{l}\text { Smoking } \\
\text { initiation a }\end{array}$ & $\begin{array}{l}776 \text { hombres } \\
\text { esquizofrénicos } \\
\text { internados y } 560 \text { controles }\end{array}$ & $\begin{array}{l}\text { Esquizofrénicos tuvieron mayor } \\
\text { prevalencia del tabaquismo }(75,8 \% \text { vs. }\end{array}$ \\
\hline
\end{tabular}


schizophrenia: a replication study in a Chinese Han Population (2010) $^{(29)}$ fueron investigados a respecto del inicio del tabaquismo, datos demográficos, médicas/psicológicas. Fagerstrom y tuvieron el índice de monóxido de carbono expiratorio mensurado.
Contestaron el Examen de

$53,9 \%$ de los controles) y de grado elevado de dependencia nicotínica $(61,4 \%$ vs. $31,4 \%$ de los controles). $72,8 \%$ empezaron a fumar antes del inicio de la esquizofrenia, $5,2 \%$ en el inicio y $22 \%$ después del inicio. Esquizofrénicos corren riesgo elevado de hacerse fumadores en la adolescencia, especialmente en los cinco años que anteceden la enfermedad.
Risk/reward decision making in schizophrenia: a preliminary examination of the influence of tobacco smoking and relationship to Wisconsin Card Sorting Task performance $(2009)^{(30)}$

\section{Tobacco} smoking behaviors in bipolar disorder: a comparison of the general population, schizophrenia and major depression (2009) $^{(31)}$

Nicotine dependence and psychiatric disorders among young males in Singapore (2009) (32)

Citation bias in reported smoking prevalence in people with schizophrenia $(2009)^{(33)}$ Does the degree of smoking effect the severity of
Fueron investigados el perfil sociodemográfico y clínico de 61 sujetos (10 no psiquiátricos y no fumadores, 9 no psiquiátricos fumadoress, 10 esquizofrénicos no fumadoress y 32 esquizofrénicos fumadores). Las funciones neuropsicológicas fueron evaluadas por medio de exámenes.
454 pacientes psiquiátricos $(99$ con trastorno bipolar, 258 esquizofrénicos y 67 con depresión grave) y 402 controles sin trastornos fueron tabaquismo por medio de procedimientos estructurados. investigados al respecto del
La prevalencia del tabaquismo fue superior en los esquizofrénicos (74\%), seguida por los portadores de trastorno bipolar (66\%) y por los portadores de depresión grave (57\%).
Fueron investigados 9702 jóvenes del sexo masculino de Cingapura sobre el tabaquismo y trastornos mentales.
Los esquizofrénicos tuvieron desempeño inferior en los exámenes neuropsicológicos si son comparados con los sujetos no psiquiátricos. El tabaquismo se asoció a la mejora en el examen que evalúa riesgo/rendimiento de toma de decisiones en los esquizofrénicos del sexo femenino.
$12,3 \%$ de los sujetos tenían dependencia nicotínica. La prevalencia de trastornos esquizoafectivos y esquizofrenia en los sujetos con dependencia nicotínica fue de 14,3\% contra $2 \%$ en los sujetos sin dependencia.

Aumento de $10 \%$ en la prevalencia del tabaquismo en la esquizofrenia fue asociado al aumento de $61 \%$ en el índice de mención del artículo.

$75 \%$ de los esquizofrénicos eran fumadores. No hubo diferencia en la prevalencia de tabaquismo al 


\begin{tabular}{|c|c|c|}
\hline $\begin{array}{l}\text { tardive } \\
\text { dyskinesia? A } \\
\text { longitudinal } \\
\text { clinical trial } \\
(2009){ }^{(34)}\end{array}$ & $\begin{array}{l}\text { tratados con } r \text { neurolépticos } \\
\text { típicos (NT), fueron investigados } \\
\text { con relación a los movimientos } \\
\text { involuntarios, ríntomas } \\
\text { negativos y positivos y } \\
\text { concentración plasmática de } \\
\text { antipsicótico. }\end{array}$ & $\begin{array}{l}\text { comparar los dos grupos (CLZ y NT). } \\
\text { Severidad aumentada de la discinesia } \\
\text { tardía fue asociada al mayor número } \\
\text { de cigarrillos/día y más tiempo de } \\
\text { tabaquismo. }\end{array}$ \\
\hline $\begin{array}{l}\text { Self-reported } \\
\text { motivation to } \\
\text { smoke in } \\
\text { schizophrenia is } \\
\text { related to } \\
\text { antipsychotic } \\
\text { drug treatment } \\
(2008) \text { (35) }\end{array}$ & $\begin{array}{l}61 \text { esquizofrénicos internados y } \\
33 \text { controles no psiquiátricos } \\
\text { fueron investigados a respecto } \\
\text { de la motivación para fumar y el } \\
\text { Examen de Fagerstrom. }\end{array}$ & $\begin{array}{l}\text { La dependencia nicotínica fue más } \\
\text { grande en los esquizofrénicos tratados } \\
\text { con antipsicóticos típicos de que en } \\
\text { aquelllos tratados con antipsicóticos } \\
\text { atípicos. La mayor motivación del } \\
\text { tabaquismo para los esquizofrénicos } \\
\text { es el placer de fumar y la necesidad de } \\
\text { estimulación psicomotora. }\end{array}$ \\
\hline $\begin{array}{l}\text { The association } \\
\text { between } \\
\text { schizophrenia } \\
\text { and smoking: } \\
\text { unexplained by } \\
\text { either the illness } \\
\text { or the prodomal } \\
\text { period (2008) }\end{array}$ & $\begin{array}{l}805 \text { sujetos }(258 \\
\text { esquizofrénicos, } 166 \text { portadores } \\
\text { de trastorno de humor y } 381 \\
\text { controles) fueron investigados } \\
\text { con relación a la historia del } \\
\text { tabaquismo. }\end{array}$ & $\begin{array}{l}74 \% \text { de los esquizofrénicos, } 62 \% \text { de } \\
\text { los trastornos de humor y } 26 \% \text { de los } \\
\text { controles eran fumadores. Mientras en } \\
\text { el grupo control el riesgo de hacerse } \\
\text { fumador disminuyó después de los } 20 \\
\text { años, no sucedió lo mismo con los } \\
\text { esquizofrénicos, que tuvieron riesgo } \\
\text { aumentado después de esa edad. }\end{array}$ \\
\hline $\begin{array}{l}\text { Tobacco } \\
\text { smoking in } \\
\text { Egyptian } \\
\text { Schizophrenia } \\
\text { patients with and } \\
\text { without } \\
\text { obsessive- } \\
\text { compulsive } \\
\text { symptoms (2007) } \\
\text { (37) }\end{array}$ & $\begin{array}{lr}87 & \text { esquizofrénicos } \\
\text { ambulatoriales fueron } \\
\text { investigados con relación al } \\
\text { tabaquismo, síntomas } \\
\text { obsesivos-compulsivos r y } \\
\text { concentración plasmática de } \\
\text { cotinina. Respondieron al } \\
\text { Examen de Fagerstrom. }\end{array}$ & $\begin{array}{l}\text { La prevalencia del tabaquismo fue } \\
\text { elevada tanto en los esquizofrénicos } \\
\text { con síntomas obsesivos-compulsivos } \\
\text { ( } 76,5 \%) \text { como en los esquizofrénicos } \\
\text { sin esos síntomas (83\%). La hipótesis } \\
\text { de que el tabaquismo es menor en los } \\
\text { esquizofrénicos con síntomas } \\
\text { obsesivos-compulsivos no fue } \\
\text { confirmada. }\end{array}$ \\
\hline $\begin{array}{l}\text { Cigarette } \\
\text { smoking in } \\
\text { Chinese male } \\
\text { inpatients with } \\
\text { schizophrenia: a } \\
\text { cross-sectional } \\
\text { analysis (2007) } \\
(38)\end{array}$ & $\begin{array}{l}279 \text { esquizofrénicos del sexo } \\
\text { masculino internados fueron } \\
\text { investigados con relación a los } \\
\text { datos demográficos, historia del } \\
\text { tabaquismo, curso de la } \\
\text { esquizofrenia. }\end{array}$ & $\begin{array}{l}40,1 \% \text { de los esquizofrénicos eran } \\
\text { tabaquistas. Los fumadores } \\
\text { presentaron grado de escolaridad } \\
\text { inferior al de los no fumadores. Los } \\
\text { fumadores presentaron más episodios } \\
\text { de intensificación de la esquizofrenia } \\
\text { de que los no fumadores. }\end{array}$ \\
\hline $\begin{array}{l}\text { Characteristics of } \\
\text { smokers with a } \\
\text { psychotic } \\
\text { disorder and } \\
\text { implications for } \\
\text { smoking } \\
\text { interventions } \\
(2007)^{(39)}\end{array}$ & $\begin{array}{l}298 \text { fumadores psicóticos, } \\
\text { residentes en la comunidad, } \\
\text { fueron investigados con relación } \\
\text { a los datos demográficos, } \\
\text { clínicos, tabaquismo, grado de } \\
\text { dependencia, etapas de cambio, } \\
\text { razones para fumar y para dejar } \\
\text { el tabaquismo. }\end{array}$ & $\begin{array}{l}56,7 \% \text { de los tfumadoress tenían } \\
\text { diagnóstico de esquizofrenia o } \\
\text { trastorno esquizoafectivo, razones } \\
\text { para fumar entre los esquizofrénicos: } \\
\text { reducción del estrese y aumento de la } \\
\text { estimulación psicomotora. }\end{array}$ \\
\hline
\end{tabular}




\begin{tabular}{|c|c|c|}
\hline $\begin{array}{l}\text { Nicotine use and } \\
\text { its correlates in } \\
\text { patients with } \\
\text { psychosis (2007) } \\
(40)\end{array}$ & 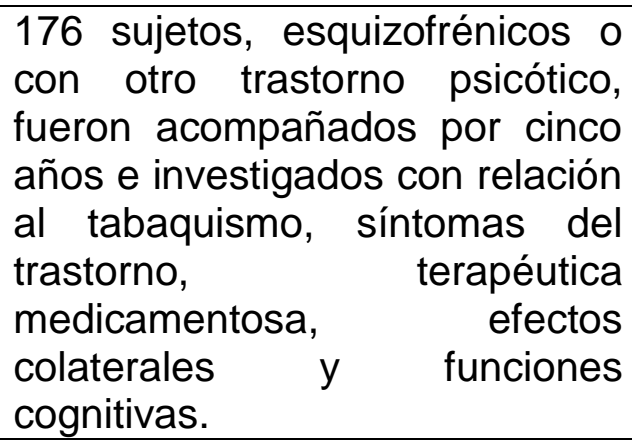 & $\begin{array}{l}\text { La prevalencia del tabaquismo en los } \\
\text { esquizofrénicos u otros trastornos } \\
\text { psicóticos relacionados con la } \\
\text { esquizofrenia fue del } 70 \% \text {. El } \\
\text { tabaquismo en los esquizofrénicos } \\
\text { está asociado a la impulsividad y a la } \\
\text { búsqueda de estímulo. }\end{array}$ \\
\hline $\begin{array}{l}\text { Subjective } \\
\text { effects and the } \\
\text { main reason for } \\
\text { smoking in } \\
\text { outpatients with } \\
\text { schizophrenia: a } \\
\text { case control } \\
\text { study (2007) }\end{array}$ & $\begin{array}{l}173 \text { esquizofrénicos y } 100 \\
\text { controles fueron investigados } \\
\text { con relación a los efectos } \\
\text { subjetivos del tabaquismo y la } \\
\text { principal razón para fumar. } \\
\text { Respondieron el Examen de } \\
\text { Fagerstrom. }\end{array}$ & $\begin{array}{l}\text { Esquizofrénicos relataron efectos } \\
\text { subjetivos del tabaquismo con mayor } \\
\text { frecuencia de que los controles. } \\
\text { Motivos para fumar entre los } \\
\text { esquizofrénicos: } 75 \% \text { fuman para } \\
\text { calmarse, } 49 \% \text { para mejorar el humor, } \\
43 \% \text { para estar más alerta, } 33 \% \text { para } \\
\text { mejorar la concentración. }\end{array}$ \\
\hline $\begin{array}{l}\text { Smoking is } \\
\text { associated with } \\
\text { schizophrenia, } \\
\text { but not with } \\
\text { mood disorders, } \\
\text { within a } \\
\text { population with } \\
\text { low smoking } \\
\text { rates (2006) }\end{array}$ & $\begin{array}{l}184 \text { colombianos, siendo } 73 \\
\text { esquizofrénicos/esquizoafectivos } \\
\text { y } 111 \text { con trastornos de humor, } \\
\text { pareados con dos controles de } \\
\text { la población general, e } \\
\text { investigados con relación a la } \\
\text { prevalencia del tabaquismo. }\end{array}$ & $\begin{array}{l}\text { La prevalencia del tabaquismo en los } \\
\text { esquizofrénicos/esquizoafetcivos fue } \\
\text { de } 26 \% \text { y en los controles de } 10 \% \text {. La } \\
\text { asociación entre tabaquismo y } \\
\text { esquizofrenia es evidente hasta } \\
\text { incluso en poblaciones con índices } \\
\text { reducidos de tabaquismo. }\end{array}$ \\
\hline $\begin{array}{l}\text { Relationship } \\
\text { between } \\
\text { obsessive- } \\
\text { compulsive } \\
\text { symptoms and } \\
\text { smoking habits } \\
\text { amongst } \\
\text { schizophrenic } \\
\text { patients (2006) } \\
\text { (43) }\end{array}$ & $\begin{array}{l}66 \\
\text { squizofrénicos/esquizoafectivos } \\
\text { fueron divididos en dos grupos, } \\
\text { de acuerdo con la puntuación en } \\
\text { la escala de síntomas obsesivo- } \\
\text { compulsivos: G1 }-47 \text { sujetos } \\
\text { con puntuación <16 y G2- } 19 \\
\text { sujetos con puntuación >16. Los } \\
\text { dos grupos fueron comparados. }\end{array}$ & $\begin{array}{lr}62 \% & \text { de } \\
\text { esquizofrénicos/esquizoafectivos eran } \\
\text { tfumadores. No hubo diferencia en la } \\
\text { prevalencia del tabaquismo entre los } \\
\text { grupos de esquizofrénicos con } \\
\text { síntomas obsesivo-compulsivos y } \\
\text { esquizofrénicos sin esos síntomas. }\end{array}$ \\
\hline $\begin{array}{l}\text { Predictors of } \\
\text { tobacco use } \\
\text { among persons } \\
\text { with mental } \\
\text { illnesses in a } \\
\text { statewide } \\
\text { population } \\
(2006)^{(44)}\end{array}$ & $\begin{array}{l}\text { Fueron consultados los registros } \\
\text { de } 111.984 \text { portadores de } \\
\text { trastornos mentales atendidos } \\
\text { por el sistema público de salud } \\
\text { mental de Colorado. }\end{array}$ & $\begin{array}{l}61,5 \% \text { de los esquizofrénicos eran } \\
\text { fumadores, la mayor prevalencia entre } \\
\text { los trastornos mentales investigados. }\end{array}$ \\
\hline $\begin{array}{l}\text { Cigarette } \\
\text { smoking in long } \\
\text { term } \\
\text { schizophrenia } \\
(2006)\end{array}$ & $\begin{array}{l}760 \text { esquizofrénicos, residentes } \\
\text { en la comunidad, investigados } \\
\text { en relación a los datos } \\
\text { sociodemográficos, historia del } \\
\text { tabaquismo, terapéutica } \\
\text { medicamentosa y curso de la } \\
\text { esquizofrenia. }\end{array}$ & $\begin{array}{l}60,5 \% \text { de los esquizofrénicos eran } \\
\text { tfumadoress, con mayor prevalencia } \\
\text { en los hombres que en las mujeres. El } \\
\text { tabaquismo fue asociado a poco nivel } \\
\text { de escolaridad, estado civil divorciado/ } \\
\text { separado, tiempo del trastorno entre } \\
10 \text { y } 19 \text { años, altas dosis de }\end{array}$ \\
\hline
\end{tabular}




\begin{tabular}{|c|c|c|}
\hline & & $\begin{array}{l}\text { neurolépticos } \\
\text { institucionales. }\end{array}$ \\
\hline $\begin{array}{l}\text { Smoking and } \\
\text { schizophrenia: is } \\
\text { symptom profile } \\
\text { related to } \\
\text { smoking and } \\
\text { which } \\
\text { antipsychotic } \\
\text { medication is of } \\
\text { benefit in } \\
\text { reducing } \\
\text { cigarette (2006) } \\
(46)\end{array}$ & $\begin{array}{l}146 \text { esquizofrénicos } \\
\text { investigados en relación a la } \\
\text { historia del tabaquismo, } \\
\text { síntomas positivos y negativos, } \\
\text { efectos extrapiramidales y } \\
\text { monóxido de carbono } \\
\text { expiratorio. }\end{array}$ & $\begin{array}{l}79 \% \text { de los esquizofrénicos eran } \\
\text { tabaquistas. Antipsicóticos típicos } \\
\text { fueron asociados con más severidad al } \\
\text { tabaquismo de que los antipsicóticos } \\
\text { atípicos. Los tabaquistas presentaron } \\
\text { menos acatisia. }\end{array}$ \\
\hline $\begin{array}{l}\text { Increased } \\
\text { nicotine and } \\
\text { cotinine levels in } \\
\text { smokers with } \\
\text { schizophrenia } \\
\text { and } \\
\text { schizoaffective } \\
\text { disorders is not a } \\
\text { metabolic effect } \\
(2005)(47)\end{array}$ & $\begin{array}{l}170 \text { tabaquistas: } 115 \\
\text { esquizofrénicos/esquizoafectivos } \\
\text { (G1- } 44 \text { no motivados a dejar de } \\
\text { fumar y G2 }-56 \text { motivados) y } 55 \\
\text { controles. Fueron investigados } \\
\text { en relación a la historia del } \\
\text { tabaquismo, datos demográficos } \\
\text { y medicamentosos, motivación } \\
\text { para dejar de fumar, grado de } \\
\text { dependencia nicotínica (Examen } \\
\text { de Fagerstrom), índice de } \\
\text { monóxido de carbono expiratorio } \\
\text { y concentración sanguínea de } \\
\text { nicotina y cotinina. }\end{array}$ & $\begin{array}{l}\text { Los niveles séricos de nicotina y } \\
\text { cotinina fueron superiores en los } \\
\text { esquizofrénicos/esquizoafectivos en } \\
\text { relación a los controles. Fue } \\
\text { confirmada la hipótesis de que los } \\
\text { esquizofrénicos captan una cantidad } \\
\text { más grande de nicotina por cigarrillo } \\
\text { que los fumadores sin trastornos } \\
\text { mentales. }\end{array}$ \\
\hline $\begin{array}{l}\text { Smoking } \\
\text { initiation and } \\
\text { schizophrenia: a } \\
\text { replication study } \\
\text { in a Spanish } \\
\text { sample (2005) } \\
(48)\end{array}$ & $\begin{array}{l}250 \text { esquizofrénicos, residentes } \\
\text { en la comunidad, y } 290 \\
\text { controles fueron investigados } \\
\text { con relación al tabaquismo, } \\
\text { dependencia nicotínica (Examen } \\
\text { de Fagerstrom) y curso del } \\
\text { trastorno psiquiátrico. }\end{array}$ & $\begin{array}{l}69 \% \text { de los esquizofrénicos y } 35 \% \text { de } \\
\text { los controles eran fumadoress. La } \\
\text { dependencia nicotínica fue más } \\
\text { elevada en los esquizofrénicos. } \\
\text { Después de los } 20 \text { años de edad, los } \\
\text { esquizofrénicos tuvieron más } \\
\text { incidencia de tabaquismo de que el } \\
\text { grupo control. }\end{array}$ \\
\hline $\begin{array}{l}\text { Cigarette } \\
\text { smoking } \\
\text { topography in } \\
\text { smokers with } \\
\text { schizophrenia } \\
\text { and matched } \\
\text { non-psychiatric } \\
\text { controls (2005) } \\
(49)\end{array}$ & $\begin{array}{l}20 \\
\text { esquizofrénicos/esquizoafectivo } \\
\text { fumadores y } 20 \text { controles } \\
\text { pareados fueron investigados } \\
\text { con relación a la concentración } \\
\text { de monóxido de carbono } \\
\text { expiratorio, concentración de } \\
\text { cotinina en la saliva y } \\
\text { dependencia nicotínica (Examen } \\
\text { de Fagerstrom). }\end{array}$ & $\begin{array}{l}\text { Los esquizofrénicos tragaron más que } \\
\text { los controles y tuvieron menor tiempo } \\
\text { de intervalo entre las tragadas. Los } \\
\text { esquizofrénicos tratados con } \\
\text { antipisicóticos típicos fumaban más } \\
\text { cigarrillos/día de que los tratados con } \\
\text { antipsicóticos atípicos, de manera que } \\
\text { también presentaron mayor grado de } \\
\text { dependencia nicotínica. }\end{array}$ \\
\hline $\begin{array}{l}\text { Smoking } \\
\text { behavior in } \\
\text { persons with a } \\
\text { schizophrenia- } \\
\text { spectrum } \\
\text { disorder: a }\end{array}$ & $\begin{array}{l}\text { Investigación cualitativa. } \\
\text { Realizadas entrevistas con } 12 \\
\text { participantes: seis recientemente } \\
\text { hospitalizados debido al primer } \\
\text { episodio psicótico y seis } \\
\text { esquizofrénicos crónicos. }\end{array}$ & $\begin{array}{l}\text { Principales ventajas del tabaquismo } \\
\text { relatadas: alivio de la ansiedad y } \\
\text { mejoría de los síntomas negativos. El } \\
\text { perjuicio financiero fue reconocido por } \\
\text { los equizofrénicos debido a la compra } \\
\text { de cigarrillos. El cese del tabaquismo }\end{array}$ \\
\hline
\end{tabular}




\begin{tabular}{|c|c|c|}
\hline $\begin{array}{l}\text { qualitative } \\
\text { investigation of } \\
\text { the } \\
\text { transtheoretical } \\
\text { model }(2005)^{(50)}\end{array}$ & & $\begin{array}{l}\text { se ve dificultado por la influencia de los } \\
\text { familiares y amigos que fuman o que } \\
\text { aprueban el acto de fumar. }\end{array}$ \\
\hline $\begin{array}{l}\text { Poor premorbid } \\
\text { school } \\
\text { performance is } \\
\text { associated with } \\
\text { later cigarette } \\
\text { smoking among } \\
\text { schizophrenia } \\
\text { patients (2005) } \\
(51)\end{array}$ & $\begin{array}{l}67 \text { esquizofrénicos de Finlandia } \\
\text { fueron investigados con relación } \\
\text { al perfil sociodemográfico, } \\
\text { tabaquismo y rendimiento } \\
\text { escolar. }\end{array}$ & $\begin{array}{l}48 \% \text { de los esquizofrénicos eran } \\
\text { tfumadoress. } \\
\text { Los fumadores presentaron peor } \\
\text { redimiento escolar que los no } \\
\text { fumadores. El bajo rendimiento escolar } \\
\text { está asociado a la disfunción en la } \\
\text { neurotransmisión nicotinérgica, que } \\
\text { puede ser compensada por el } \\
\text { tabaquismo. }\end{array}$ \\
\hline $\begin{array}{l}\text { Is initiation of } \\
\text { smoking } \\
\text { associated with } \\
\text { the prodromal } \\
\text { phase of } \\
\text { schizophrenia? } \\
(2005){ }_{(52)}\end{array}$ & $\begin{array}{l}100 \text { esquizofrénicos, } 55 \text { con } \\
\text { otras psicoses, } 315 \text { con } \\
\text { trastornos no psicóticos y } 10.464 \\
\text { controles fueron investigados, a } \\
\text { partir de los registros de } \\
\text { Finlandia, con relación al início } \\
\text { del trastorno y otras variables } \\
\text { asociadas al tabaquismo. }\end{array}$ & $\begin{array}{l}48 \% \text { de los esquizofrénicos eran } \\
\text { tabaquistas contra } 29 \% \text { de los } \\
\text { controles. La edad de inicio de la } \\
\text { esquizofrenia fue próxima a la edad } \\
\text { del inicio del tabaquismo, con una } \\
\text { media de diferencia de } 2,3 \text { años. El } \\
\text { tabaquismo puede ser un indicador de } \\
\text { la fase prodrómica de la esquizofrenia. }\end{array}$ \\
\hline $\begin{array}{l}\text { Higher rates of } \\
\text { cigarette } \\
\text { smoking in male } \\
\text { adolescents } \\
\text { before the onset } \\
\text { of schizophrenia: } \\
\text { a historical- } \\
\text { prospective } \\
\text { cohort study } \\
(2004){ }_{(53)}\end{array}$ & $\begin{array}{l}\text { Fueron investigados } 14.248 \\
\text { jóvenes en Israel, sin diagnósico } \\
\text { de trastorno mental en el } \\
\text { momento de la selección en el } \\
\text { servicio militar. Tuvieron historia } \\
\text { de internación psiquiátrica } \\
\text { investigada por un período de } \\
\text { acompañamiento de 4-16 años. }\end{array}$ & $\begin{array}{l}28,4 \% \text { de los sujetos relataron fumar al } \\
\text { menos um cigarrillo/día. En los } 4-16 \\
\text { años de acompañamiento la } \\
\text { prevalencia de esquizofrenia fue de } \\
0,3 \%(n=44) \text {. El tabaquismo en la } \\
\text { juventud fue considerado factor de } \\
\text { riesgo para la esquizofrenia. }\end{array}$ \\
\hline $\begin{array}{l}\text { Stages of } \\
\text { change in } \\
\text { smokers with } \\
\text { schizophrenia or } \\
\text { schizoaffective } \\
\text { disorder and in } \\
\text { the general } \\
\text { population } \\
(2004)^{(54)}\end{array}$ & $\begin{array}{l}\text { Fueron investigados } 251 \\
\text { esquizofrénicos/esquizoafectivos } \\
\text { ambulatoriales y } 742 \text { controles } \\
\text { de la población general en } \\
\text { relación al perfil } \\
\text { sociodemográfico, historia del } \\
\text { tabaquismo y etapas de cambio. }\end{array}$ & $\begin{array}{l}70 \% \text { de } \\
\text { esquizofrénicos/esquizoafectivos y } \\
28 \% \text { de los controles eran fumadores. } \\
\text { Etapas motivacionales para dejar de } \\
\text { fumar en los esquizofrénicos: } 79 \% \text { pre } \\
\text { contemplación, } 18 \% \text { contemplación, } \\
3 \% \text { preparación. Una minoría de } \\
\text { esquizofrénicos/esquizoafectivos está } \\
\text { motivada a dejar de fumar. }\end{array}$ \\
\hline $\begin{array}{l}\text { Nicotine use in } \\
\text { schizophrenia } \\
\text { and disinhibition } \\
(2004)\end{array}$ & $\begin{array}{l}100 \text { esquizofrénicos fueron } \\
\text { investigados sobre síntomas } \\
\text { positivos y negativos, } \\
\text { impulsividad, búsqueda de } \\
\text { sensaciones y dependencia } \\
\text { nicotínica. }\end{array}$ & $\begin{array}{l}67 \% \text { de } \\
\text { esquizofrénicos/esquizoafectivos eran } \\
\text { tfumadoress. La puntuación en la } \\
\text { escala de búsqueda de sensaciones } \\
\text { fue superior en los fumadores. El } \\
\text { tabaquismo puede ayudar a normalizar } \\
\text { la inhibición en esquizofrénicos. }\end{array}$ \\
\hline $\begin{array}{l}\text { Cigarette } \\
\text { smoking among } \\
\text { patients with }\end{array}$ & $\begin{array}{l}\text { Fueron evaluados } 144 \\
\text { esquizofrénicos o bipolares y } \\
144 \text { controles saludables con }\end{array}$ & $\begin{array}{l}57,5 \% \text { de los esquizofrénicos, } 55,1 \% \\
\text { de los bipolares y } 47,3 \% \text { de los } \\
\text { controles eran tfumadores. El }\end{array}$ \\
\hline
\end{tabular}




\begin{tabular}{|c|c|c|}
\hline $\begin{array}{l}\text { ophrenia } \\
\text { ipolar } \\
\text { lers (2004) }\end{array}$ & $\begin{array}{l}\text { relación al tabaquismo } \\
\text { síntomas psiquiátricos. }\end{array}$ & $\begin{array}{l}\text { tabaquismo está asociado a la mayor } \\
\text { puntuación en la escala de síntomas } \\
\text { positivos entre los esquizofrénicos. }\end{array}$ \\
\hline $\begin{array}{l}\text { Reward value of } \\
\text { cigarette } \\
\text { smoking for } \\
\text { comparably } \\
\text { heavy smoking } \\
\text { schizophrenic, } \\
\text { depressed and } \\
\text { nonpatient } \\
\text { smokers (2003) } \\
(57)\end{array}$ & $\begin{array}{l}26 \text { esquizofrénicos, } 26 \\
\text { depresivos y } 26 \text { controles fueron } \\
\text { investigados en relación al } \\
\text { tabaquismo, compromiso en } \\
\text { actividades placerosas, ventajas } \\
\text { y desventajas percibidas del } \\
\text { tabaquismo y dependencia } \\
\text { nicotínica (Examen de } \\
\text { Fagerstrom). }\end{array}$ & $\begin{array}{l}\text { Tanto los esquizofrénicos como los } \\
\text { depresivos eligieron el tabaquismo } \\
\text { como su actividad favorita con mayor } \\
\text { frecuencia que los fumadores no } \\
\text { psiquiátricos. Los esquizofrénicos y los } \\
\text { depresivos perciben más beneficios } \\
\text { del tabaquismo que los sujetos no } \\
\text { psiquiátricos. }\end{array}$ \\
\hline $\begin{array}{l}\text { Investigating the } \\
\text { association } \\
\text { between } \\
\text { cigarette } \\
\text { smoking and } \\
\text { schizophrenia in } \\
\text { a cohort study } \\
(2003)(58)\end{array}$ & $\begin{array}{l}50.087 \text { reclutas suecos fueron } \\
\text { acompañados por medio de } \\
\text { registros nacionales } \\
\text { internación, en el periodo de } \\
1970 \text { a 1996, para investigar la } \\
\text { ocurrencia de ingreso por } \\
\text { esquizofrenia. }\end{array}$ & $\begin{array}{l}\text { El tabaquismo en la franja etaria de los } \\
18-20 \text { años fue asociado al menor } \\
\text { índice de desarrollo de la } \\
\text { esquizofrenia. Los autores sugieren } \\
\text { que el tabaquismo puede actuar como } \\
\text { factor protector en contra del } \\
\text { desarrollo de la esquizofrenia. }\end{array}$ \\
\hline $\begin{array}{l}\text { Smoking and } \\
\text { substance abuse } \\
\text { in outpatients } \\
\text { with } \\
\text { schizophrenia: a } \\
2 \text { year follow up } \\
\text { study in Turkey } \\
(2003) \text { (59) }\end{array}$ & $\begin{array}{lr}136 & \text { esquizofrénicos } \\
\text { ambulatoriales fueron } \\
\text { investigados con relación al } \\
\text { tabaquismo, curso de la } \\
\text { enfermedad y terapéutica } \\
\text { medicamentosa. r rueron } \\
\text { entrevistados en intervalos } \\
\text { regulares por un período de dos } \\
\text { años. }\end{array}$ & $\begin{array}{l}50 \% \text { de los esquizofrénicos eran } \\
\text { fumadores. Los fumadores estaban } \\
\text { recibiendo dosis diarias de } \\
\text { neurolépticos más elevadas de que los } \\
\text { no fumadores. }\end{array}$ \\
\hline $\begin{array}{l}\text { Smoking habits } \\
\text { in Japanese } \\
\text { patients with } \\
\text { schizophrenia } \\
(2003)(60)\end{array}$ & $\begin{array}{l}137 \text { japoneses esquizofrénicos } \\
\text { acompañados en ambulatorio } \\
\text { fueron investigados con relación } \\
\text { al perfil sociodemográfico, } \\
\text { tabaquismo, historia de la } \\
\text { enfermedad y terapéutica } \\
\text { medicamentosa. }\end{array}$ & $\begin{array}{l}34 \% \text { de los esquizofrénicos y } 37 \% \text { de } \\
\text { la población de Japón eran fumadores. } \\
\text { Variables asociadas al tabaquismo en } \\
\text { la esquizofrenia: sexo masculino, } \\
\text { internaciones psiquiátricas y consumo } \\
\text { de alcohol. }\end{array}$ \\
\hline $\begin{array}{l}\text { Schizophrenia } \\
\text { and tobacco } \\
\text { smoking: a } \\
\text { replication study } \\
\text { in another US } \\
\text { psychiatric } \\
\text { hospital (2002) } \\
(61)\end{array}$ & $\begin{array}{l}588 \text { pacientes psiquiátricos } \\
\text { internados fueron investigados } \\
\text { con relación a la cuantidad de } \\
\text { cigarrillo fumado/día, durante la } \\
\text { internación psiquiátrica. }\end{array}$ & $\begin{array}{l}75 \% \text { de los esquizofrénicos eran } \\
\text { fumadores. La prevalencia fue mayor } \\
\text { en los esquizofrénicos del sexo } \\
\text { masculino. }\end{array}$ \\
\hline $\begin{array}{l}\text { Smoking in } \\
\text { schizophrenia - } \\
\text { all is not } \\
\text { biological (2002) } \\
\text { (62) }\end{array}$ & $\begin{array}{l}687 \text { sujetos: } r \\
\text { esquizofrénicos, } 84 \\
\text { trastornos de humor mayor, } 140 \\
\text { con trastornos no psicóticos y } \\
177 \text { sin trastornos mentales. }\end{array}$ & $\begin{array}{l}38 \% \text { de los esquizofrénicos, } 24 \% \\
\text { trastorno de humor y } 23 \% \text { controles } \\
\text { eran fumadores. Variables asociadas } \\
\text { al tabaquismo en la esquizofrenia: } \\
\text { soltero, desempleo y bajo status }\end{array}$ \\
\hline
\end{tabular}




\begin{tabular}{|c|c|c|}
\hline & $\begin{array}{l}\text { Fueron investigados en relación } \\
\text { al perfil sociodemográfico, } \\
\text { tratamiento medicamentoso e } \\
\text { historia del tabaquismo. }\end{array}$ & $\begin{array}{l}\text { económico. } 40 \% \text { de los } \\
\text { esquizofrénicos iniciaron en el } \\
\text { tabaquismo después del inicio del } \\
\text { trastorno. } 45 \% \text { de los esquizofrénicos } \\
\text { no sienten ningún efecto con el uso del } \\
\text { cigarrillo, } 34 \% \text { disminuyen la tensión y } \\
14 \% \text { relataron fumar para mejorar el } \\
\text { proceso cognitivo y para sentirse parte } \\
\text { del mundo. }\end{array}$ \\
\hline $\begin{array}{l}\text { Initiation of daily } \\
\text { smoking and } \\
\text { nicotine } \\
\text { dependence in } \\
\text { schizophrenia } \\
\text { and mood } \\
\text { disorders (2002) } \\
\text { (63) }\end{array}$ & $\begin{array}{l}\text { Participaron } 66 \text { sujetos con } \\
\text { esquizofrenia, } 51 \text { con trastornos } \\
\text { de humor y 404 controles. } \\
\text { Respondieron el Examen de } \\
\text { Fagerstrom y tuvieron la } \\
\text { concentración de monóxido de } \\
\text { carbono mensurada. }\end{array}$ & $\begin{array}{l}\text { La prevalencia del tabaquismo en la } \\
\text { esquizofrenia fue de } 83 \% \text { contra } 65 \% \\
\text { en los trastornos de humor y } 26 \% \text { en } \\
\text { los controles. Después de los } 20 \text { años } \\
\text { de edad, la incidencia del tabaquismo } \\
\text { fue mayor en los esquizofrénicos de } \\
\text { que en los trastornos de humor y en } \\
\text { los controles. }\end{array}$ \\
\hline $\begin{array}{l}\text { Relationship } \\
\text { between tobacco } \\
\text { smoking and } \\
\text { positive and } \\
\text { negative } \\
\text { symptoms in } \\
\text { schizophrenia } \\
(2002)\end{array}$ & $\begin{array}{l}87 \text { esquizofrénicos internados } \\
\text { fueron investigados por medio } \\
\text { de instrumentos estructurados } \\
\text { que evaluan la dependencia } \\
\text { nicotínica (Examen de } \\
\text { Fagerstrom) y los síntomas } \\
\text { positivos y negativos de la } \\
\text { esquizofrenia. }\end{array}$ & $\begin{array}{l}76 \% \text { de los esquizofrénicos tenían } \\
\text { dependencia nicotínica. Fue } \\
\text { encontrada asociación positiva entre el } \\
\text { grado de dependencia nicotínica y los } \\
\text { sintomas negativos. El tabaquismo fue } \\
\text { asociado a un mayor número de } \\
\text { internaciones psiquiátricas. }\end{array}$ \\
\hline $\begin{array}{l}\text { Factors affecting } \\
\text { smoking in } \\
\text { schizophrenia } \\
(2001)^{(65)}\end{array}$ & $\begin{array}{l}406 \text { esquizofrénicos fueron } \\
\text { investigados por medio de } \\
\text { procedimientos estructurados } \\
\text { con relación al perfil } \\
\text { sociodemográfico, tabaquismo y } \\
\text { curso de la esquizofrenia. }\end{array}$ & $\begin{array}{l}58 \% \text { de los esquizofrénicos eran } \\
\text { fumadores frente al } 42 \% \text { de los } \\
\text { controles. } 86 \% \text { de los esquizofrénicos } \\
\text { iniciaron el tabaquismo después del } \\
\text { inicio del trastorno. El tabaquismo fue } \\
\text { asociado al aumento de los síntomas } \\
\text { positivos y la disminución de los } \\
\text { negativos. }\end{array}$ \\
\hline
\end{tabular}

Cuadro II - Principales informaciones obtenidas de las revisiones de literatura/discusiones teóricas

\begin{tabular}{|l|l|}
\hline Título (año) & Síntesis \\
\hline Why do patients & Inicialmente, se creía que los esquizofrénicos fumaban \\
with schizophrenia & solamente para aumentar el metabolismo hepático y para \\
smoke? (2010) (66) & $\begin{array}{l}\text { restaurar el bloqueo dopaminérgico causado por algunos } \\
\text { antipsicóticos, mejorando los efectos colaterales de los } \\
\text { antipsicóticos. Hoy en día, se cree que el tabaquismo también } \\
\text { funciona como refuerzo positivo a la medida que mejora los } \\
\text { síntomas psiquiátricos, especialmente los negativos, envueltos } \\
\text { en el proceso de cognición. }\end{array}$ \\
\hline $\begin{array}{l}\text { Tobacco use among } \\
\text { individuals with } \\
\text { schizophrenia: what watheron de forma significativa a la }\end{array}$ & $\begin{array}{l}\text { Las industrias de tabaco contribuyeron de } \\
\text { propagación de la creencia del efecto de automedicación del } \\
\text { tabaquismo en los esquizofrénicos y a la alta prevalencia del }\end{array}$ \\
\hline
\end{tabular}




\begin{tabular}{|c|c|}
\hline $\begin{array}{l}\text { role has the tobacco } \\
\text { industry played? } \\
(2008){ }^{(67)}\end{array}$ & $\begin{array}{l}\text { baquismo hoy en día, así como la cultura del tabaquismo en las } \\
\text { stituciones psiquiátricas. }\end{array}$ \\
\hline $\begin{array}{l}\text { Smoke, smoke, } \\
\text { smoke that cigarette } \\
(2006)^{(68)}\end{array}$ & $\begin{array}{l}\text { Los autores discuten la alta prevalencia del tabaquismo en los } \\
\text { esquizofrénicos y cómo eso afecta a la economía del usuario. } \\
\text { Describen los aspectos biológicos del tabaquismo en los } \\
\text { esquizofrénicos relacionados a la dopamina: fuman para } \\
\text { compensar la baja regulación de dopamina en la corteza frontal, } \\
\text { mejorando así los perjuicios cognitivos. Discuten cómo la } \\
\text { enfermería sigue tratando esa problemática. Algunos } \\
\text { profesionales de enfermería creen que como el cigarrillo puede } \\
\text { proporcionar placer para el esquizofrénico, el tabaquismo debe } \\
\text { ser permitido. Muchos enfermeros psiquiátricos creen que los } \\
\text { beneficios breves del tabaquismo no compensan los perjuicios } \\
\text { causados a la salud y al tratamiento. }\end{array}$ \\
\hline $\begin{array}{l}\text { Comorbidades } \\
\text { psiquiátricas } \\
\text { tabagismo no } \\
\text { (69) }\end{array}$ & $\begin{array}{l}\text { La revisión de literatura realizada indica que las comorbidades } \\
\text { psiquiátricas están comúnmente asociadas al tabaquismo, de } \\
\text { modo especial los trastornos de humor, los trastornos de } \\
\text { ansiedad y la esquizofrenia. }\end{array}$ \\
\hline $\begin{array}{l}\text { A meta-analysis of } \\
\text { worldwide studies } \\
\text { demonstrates an } \\
\text { association between } \\
\text { schizophrenia and } \\
\text { tobacco smoking } \\
\text { behaviors (2005) }(70)\end{array}$ & $\begin{array}{l}\text { Fue realizado un metanálisis a partir de } 42 \text { estudios realizados en } \\
20 \text { naciones. Se ha confirmado la asociación entre tabaquismo y } \\
\text { esquizofrenia (media de prevalencia de } 62 \% \text { ). Grado elevado de } \\
\text { dependencia nicotínica fue más prevalente en los esquizofrénicos } \\
\text { de que en la población general. Hay relación entre tabaquismo y } \\
\text { esquizofrenia y esta relación es consistente entre los países. }\end{array}$ \\
\hline $\begin{array}{l}\text { Financial } \\
\text { implications of } \\
\text { cigarette smoking } \\
\text { among individuals } \\
\text { with schizophrenia } \\
(2004) \text { (71) }\end{array}$ & $\begin{array}{l}\text { aciones financieras del tabaquismo en la esquizofrenia } \\
\text { erables, pues gastan alrededor de } 30 \% \text { de su renta en } \\
\text { de cigarrillos. La sobrecarga financiera del tabaquismo } \\
\text { quizofrénicos es a menudo negligenciada. }\end{array}$ \\
\hline $\begin{array}{l}\text { The association } \\
\text { between } \\
\text { schizophrenia and } \\
\text { cigarette smoking: a } \\
\text { review of the } \\
\text { literature and } \\
\text { implications for } \\
\text { mental health } \\
\text { nursing practice } \\
(2003) \text { (72) }\end{array}$ & $\begin{array}{l}\text { La relación entre tabaquismo y esquizofrenia, especialmente en } \\
\text { los esquizofrénicos internados en servicios psiquiátricos, y las } \\
\text { explicaciones para esta asociación son discutidas, } \\
\text { concluyéndose que el tabaquismo en la esquizofrenia es } \\
\text { complejo, pues abarca procesos psicopatológicos, bioquímicos y } \\
\text { neurofarmacológicos. Es discutido cómo el enfermero psiquiátrico } \\
\text { sigue trabajando con esa temática, pues se cree que los } \\
\text { enfermeros psiquiátricos están en posición única para causar } \\
\text { impacto positivo en la vida de los esquizofrénicos fumadores; } \\
\text { llevándolos a la reflexiónr y al cambio de comportamiento. }\end{array}$ \\
\hline $\begin{array}{l}\text { Relação entre } \\
\text { tabagismo e } \\
\text { transtornos } \\
\text { psiquiátricos (2 } \\
\text { (73) }\end{array}$ & $\begin{array}{l}\text { Los autores presentaron las principales hipótesis que pueden } \\
\text { explicar el tabaquismo en la esquizofrenia: el tabaquismo ayuda } \\
\text { a disminuir los efectos colaterales, produce relajación, disminuye } \\
\text { la ansiedad y el hastío, mejora la concentración, reduce la } \\
\text { hiperestimulación, mejora los síntomas negativos y positivos, y es } \\
\text { reponsable de uno de los pocos placeres que los esquizofrénicos } \\
\text { experimentan en su día a día. }\end{array}$ \\
\hline
\end{tabular}




\section{DISCUSIÓN}

La relación entre la esquizofrenia y el tabaquismo es indiscutible en la literatura científica. La alta prevalencia del tabaquismo entre los esquizofrénicos se ha tornado característica de esta población. Los estudios analizados en esta revisión integradora han mostrado una prevalencia del tabaquismo variada, dependiendo de la muestra estudiada (13-83\%), con una media de $59 \%$.

La complejidad de la asociación entre el tabaquismo y la esquizofrenia es evidente en el gran número de estudios publicados sobre esta temática y en los diferentes enfoques que los investigadores les han dado. En general, los resultados de los estudios arriba indican la necesidad de la realización de investigaciones con diferentes perspectivas, que muestran que las posibilidades del estudio sobre el tabaquismo en la esquizofrenia parecen ser inagotables.

Los estudiosos han analizado la influencia de las industrias del tabaco en esta alta prevalencia del tabaquismo en la esquizofrenia. A través de una búsqueda en documentos secretos, obtenidos por medio de procesos disponibles en el sitio de la biblioteca virtual sobre el tabaco de la Universidad de California, los autores señalan que el interés de las industrias del tabaco por la población portadora de esquizofrenia se inició en la década de 1950 cuando algunos estudios del medio científico indicaron una baja incidencia de cáncer en los esquizofrénicos fumadores en comparación con los fumadores de la población en general $^{(67)}$.

Las industrias de tabaco aprovecharan la divulgación de la hipótesis de la resistencia al cáncer en los esquizofrénicos para incentivar el consumo del tabaco en esta población que se ha convertido, desde hace muchos años, en su población de destino en las campañas publicitarias.

Esta creencia ha prevalecido hasta la década de 1980 cuando se realizaron las búsquedas con el fin de probar la hipótesis de la protección genética contra el cáncer en los pacientes con esquizofrenia, sugiriendo que el número reducido de cáncer en los fumadores esquizofrénicos era debido a la subestimación de las causas de las muertes en esta población ${ }^{(13,67,74-76)}$.

Las industrias del tabaco han contribuido mucho, al lo largo de los años, a través de la financiación de la investigaciones, al inicio y mantenimiento del tabaquismo entre los esquizofrénicos, publicando solamente las búsquedas cuyos resultados eran favorables a sus intereses comerciales. Aquellas búsquedas que demostraban los perjuicios del tabaquismo eran archivadas ${ }^{(67)}$.

Con esa influencia del interés comerciales de las industrias del tabaco en la financiación/divulgación de muchas búsquedas científicas, las industrias del tabaco son, en parte, las responsables por las creencias de que el tabaquismo presenta efecto de automedicación en los esquizofrénicos y que la cesación del tabaquismo agrava el cuadro psiquiátrico, lo que representa una gran barrera para las estrategias de disminución de la incidencia y prevalencia del tabaquismo en esta población ${ }^{(67)}$.

Tras los estudios analizados en esta revisión integradora, es posible resumir las principales características de los esquizofrénicos fumadores: sexo masculino, solteros y divorciados, desempleados, bajo grado de escolaridad, consumo de alcohol, largo tiempo de esquizofrenia y mayor severidad, mayor ocurrencia de episodios psicóticos, mayor número 
de internaciones psiquiátricas (cuidados institucionales), desarrollo de la discinesia tardía, mejor desempeño cognitivo, elevadas dosis de agentes antipsicóticos y tratamiento con antipsicóticos típicos.

En general, los esquizofrénicos presentaron alto grado de dependencia nicotínica, mostraron obtener mayor cantidad de nicotina por cigarrillo fumado e invertir la mayor parte de su renta en la manutención del tabaquismo ${ }^{(23,24,27,29,50)}$. Algunos estudios mostraron que el inicio del tabaquismo ocurre antes de la aparición de los primeros síntomas psicóticos, acreditando que el tabaquismo puede ser considerado factor de riesgo para el desarrollo de la esquizofrenia o simplemente una señal de la fase prodrómica del trastorno ${ }^{(24,29,52)}$. Hubo estudios que mostraron que el inicio del tabaquismo ocurrió después de la manifestación clínica de la esquizofrenia ${ }^{(65)}$ y solamente un estudio ha sugerido que el tabaquismo en la adolescencia presenta efecto protector contra el desarrollo del trastorno ${ }^{(58)}$.

A pesar de las controversias en cuanto al inicio del tabaquismo y la manifestación de los primeros síntomas psicóticos, parece haber consenso en que uno de los motivos que dificulta el cese del tabaquismo entre los esquizofrénicos es la percepción de la mejoría de los síntomas negativos. Los estudiosos que evidenciaron esta asociación explican que una de las funciones de la nicotina en el sistema nervioso central es el aumento de la actividad dopaminérgica en la corteza frontal. Como los síntomas negativos (apatía, anhedonia, perjuicio en la concentración, en la memoria y en la atención, déficit motor) ocurren justamente por perjuicio en la neurotransmisión dopaminérgica frontal el tabaquismo actúa como antagonista temporario de estos síntomas ${ }^{(8,24,30,65-68,72)}$.

En relación a los síntomas positivos (delirios, alucinaciones, alteraciones en el habla, entre otros) existe una controversia respecto al efecto de la nicotina en su presentación. Los síntomas positivos ocurren debido a una hiperactividad del sistema dopaminérgico en la región mesolímbica del SNC, lo que lleva a creer que la nicotina podría agravar esos síntomas $^{(8,76)}$. Algunos estudios demuestran que el tabaquismo está asociado al aumento de los síntomas positivos ${ }^{(56,65)}$, lo que podría justificar la mayor severidad de la esquizofrenia en esa población, así como un mayor número de internaciones y dosis elevadas de neurolépticos como tentativa de contener esos síntomas.

Búsquedas demuestran que hay diferencia en el consumo del tabaco entre esquizofrénicos en uso de antipsicóticos de $1^{\underline{a}}$ generación (típicos) y de $2^{\underline{a}}$ generación (atípicos), tanto en relación a prevalencia del tabaquismo como número de cigarrillos/día y el grado de dependencia nicotínica. Los antipsicóticos de $1^{\text {a }}$ generación presentan gran impacto en los síntomas positivos, pero no interfieren en los síntomas negativos, estando su utilización asociada a la mayor prevalencia del tabaquismo, ya que la nicotina alivia los síntomas que ese tipo de antipsicótico no puede tratar. Los antipsicóticos de $2^{a}$ generación están asociados a la menor prevalencia del tabaquismo, pues actúan tanto en los síntomas positivos como en los síntomas negativos, revirtiendo la hipofrontalidad mejor que la nicotina, habiendo alivio de los síntomas negativos y disminución de la necesidad de la nicotina para tratar esos síntomas ${ }^{(35,46,49,77)}$.

Además de la mejoría de los procesos cognitivos, el tabaquismo también reduce la hiperestimulación sensorial que experimentan los esquizofrénicos como un sistema desagradable. En la esquizofrenia, la persona presenta dificultad de concentrarse solamente en un estímulo, se queda alerta a todos los estímulos, incluso los ruidos ambientales más comunes, lo que contribuye a su desatención en relación a los hechos en su entorno. La nicotina tiene un papel importante en la mejoría de la capacidad de seleccionar los estímulos 
sensoriales, lo que contribuye indirectamente en el aumento de la atención y la concentración ${ }^{(8,78,79)}$.

Una de las razones a que contribuye el tabaquismo en los pacientes con esquizofrenia es el alivio de los efectos colaterales de los medicamentos utilizados. Se cree que los productos resultantes de la combustión del tabaco inducen a las enzimas hepáticas a aumentar el metabolismo de los antipsicóticos. Luego, el esquizofrénico fumador presenta menor tasa de neurolépticos en la circulación de la sangre cuando comparados con los que no son fumadores, necesitando de dosis mayores de antipsicóticos para el control del cuadro psiquiátrico. El aumento del metabolismo de los antipsicóticos es responsable por la disminución de los efectos colaterales, especialmente los parkinsonianos y el cambio de la sintomatología psiquiátrica que se puede agravar ${ }^{(8,46,66,78)}$.

Lo que motiva a los portadores de esquizofrenia para el tabaquismo es similar a la población no psiquiátrica: alivio de la tensión y de la ansiedad y una alternativa para ocupar el tiempo sin actividad. En el caso del esquizofrénico, el tabaquismo se torna más importante, pues facilita algunas situaciones que son sinónimos de limitaciones en la esquizofrenia como la interacción social y la obtención de placer y satisfacción. Autores defienden que el tabaquismo puede ayudar a soportar los síntomas psiquiátricos, aumentar la estimulación psicomotora, mejorar el afecto negativo, mejorar la concentración y ayudar a la persona a sentirse parte del mundo ${ }^{(8,23,27,35,39,41,50,62,72)}$.

Por medio de esta revisión integradora, es posible ver que la literatura científica ha mostrado una preocupación creciente con el tabaquismo en la esquizofrenia. Sin embargo, se ha hablado poco sobre cómo la enfermería puede actuar con esa realidad.

La dificultad de la acción de la enfermería ante esta problemática puede ser un reflejo histórico de su inserción en las instituciones psiquiátricas, pues, a lo largo de los años, el tabaquismo fue utilizado como una de las "herramientas" del cuidado de enfermería en esos servicios. El cigarrillo circulaba libremente en muchas unidades, incluso siendo utilizado por los profesionales de los grupos. Era ofrecido a los pacientes internados a fin de controlar el comportamiento, prevenir las actitudes agresivas e incentivar la adhesión al tratamiento medicamentoso, ideas hasta hoy presentes en las prácticas ${ }^{(8,10,13,80)}$.

Se cree que la enfermería, a través del cuidado directo, del relacionamiento terapéutico, del abordaje comprensivo, de su capacidad de comunicación con los demás miembros del equipo multidisciplinar y de su participación en la elaboración del proyecto terapéutico individualizado puede ayudar al esquizofrénico fumador a encontrar recursos para enfrentar las limitaciones del trastorno mental y de la dependencia nicotínica ${ }^{(7-13)}$.

En el cuidado de enfermería en psiquiatría algunas acciones son importantes en el abordaje de los fumadores como el conocimiento de la historia del tabaquismo (tentativas anteriores para parar de fumar, motivos de recaídas, recursos encontrados para superar los periodos de abstinencia), del grado de dependencia nicotínica, de las motivaciones y de las barreras para parar de fumar. Ese abordaje al paciente psiquiátrico puede orientar el planeamiento del cuidado de enfermería y de los demás miembros del grupo.

Es importante también que el enfermo investigue la ocurrencia de dependencia de otros tipos de drogas y que sea hecha una evaluación física del portador del trastorno mental, de modo a identificar cambios físicos relacionados al aumento de morbilidad y mortalidad en esa población. La enfermería necesita también considerar los efectos de la abstinencia del 
tabaco que pueden ser confundidos durante la internación con los síntomas del cuadro psiquiátrico presentado ${ }^{(7-9,81)}$.

A pesar de que la enfermería ocupa una posición privilegiada en el cuidado a los pacientes psiquiátricos, que puede favorecer el abordaje del tabaquismo, ella encuentra muchas barreras en su práctica diaria que limitan sus acciones. Una de las principales barreras es la falta de apropiación de conocimiento, evidenciada en la ausencia de publicaciones nacionales respecto del asunto y en la escasez de publicaciones internacionales que priorizan la discusión de los aspectos neurobiológicos en lugar de las implicaciones y posibles intervenciones de enfermería.

Las demás barreras reconocidas son la creencia de que el paciente psiquiátrico no tiene motivación para dejar de fumar y que el cese del tabaquismo no se encuentra entre las prioridades del abordaje, creencia de que el tabaquismo favorece la relación profesionalpaciente, recelo en sacar uno de los pocos placeres que el portador del trastorno mental experimenta en su día a día, recelo en aumentar el estrés del paciente, falta de tiempo y de confiabilidad en su potencial profesional para ese tipo de abordaje, subestimando los preceptos de la profesión ${ }^{(8,10,12,71,82-84)}$.

El conocimiento al respecto del las cuestiones incluidas en la asociación entre tabaquismo y trastornos mentales es fundamental para instrumentalizar los enfermeros en la planificación de estrategias para combatir el tabaquismo, para enfrentar la cultura existente, el estigma y las percepciones personales sobre ese fenómeno que limitan el abordaje de los profesionales. El desafío es incluir el abordaje al fumador como parte del cuidado de enfermería, ofreciendo a los pacientes psiquiátricos la oportunidad de reflexionar acerca del tabaquismo en su vida y sobre su real disposición para recibir ayuda para abandonar el hábito ${ }^{(8,11,13,85)}$.

\section{CONCLUSIÓN}

La asociación del tabaquismo y la esquizofrenia es preocupante, pues a pesar de los aparentes beneficios del tabaquismos en esta población (mejora de los síntomas negativos, disminución de los efectos secundarios de los medicamentos, sentimientos de placer) interfiere en el tratamiento médico, agrava los síntomas del trastorno, mayor ocurrencia de episodios psicóticos, riesgo para la discinesia tardía y mayor vulnerabilidad para las enfermedades crónicas, pudiendo causar más sufrimiento y limitaciones.

La escasez de publicaciones desde la perspectiva de la enfermería, pone de manifiesto la falta de posicionamiento del enfermero frente a esta temática. El tabaquismo en la esquizofrenia es más un problema que requiere de los enfermeros atención y acción. Así, es necesaria la ampliación de su campo de conocimiento y que transformen el conocimiento producido en prácticas del cuidado de enfermería.

\section{Agradecimiento:}

Agradecemos al Consejo Nacional de Desarrollo Científico y Tecnológico - CNPq (Brasil) su apoyo financiero.

\section{BIBLIOGRAFÍA}

1. Brasil. Ministério da Saúde. Brasil - advertências sanitárias nos produtos de tabaco 2009. Rio de Janeiro: INCA; 2008. 59 p. 
2. World Health Organization. Who report on the global tobacco epidemic, 2009: Implementing smoke-free environments. Geneva: World Health Organization; 2009.

3. Lasser K, Boyd JW, Woolhandler S, et al. Smoking and mental illness: a population based prevalence study. Journal of American Medical Association. 2000;284(20):2606-10.

4. Brasil. Ministério da Saúde. A ratificação da Convenção-Quadro para o controle do tabaco para o Brasil: mitos e verdades. Rio de Janeiro: INCA; 2004. 34 p.

5. Hall SM, Prochaska JJ. Treatment of smokers with co-occuring disorders: emphasis on integration in mental health and addiction treatment settings. Annual Review of Clinical Psychology. 2009; 5:409-31.

6. Schneider B, Wetterling T, Georgi K, et al. Smoking differently modifies suicide risk of affective disorders, substance use disorders and social factors. Journal of Affective Disorders. 2009;112(1-3):165-73.

7. Cataldo JK. The role of advanced practice psychiatric nurses in treating tobacco use and dependence. Archives of Psychiatric Nursing. 2001;15(3):107-19.

8. McCloughen A. The association between schizophrenia and cigarette smoking: a review of the literature and implications for mental health nursing practice. International Journal of Mental Health Nursing. 2003;12(2):119-29.

9. Wallace-bell M. Smoking cessation: the case for hospital based interventions. Professional Nurse. 2003;19(3):145-8.

10. Lawn S, Condon J. Psychiatric nurses` ethical stance on cigarette smoking by patients: determinants and dilemmas in their role in supporting cessation. International Journal of Mental Health Nursing. 2006;15:111-18.

11. Rice VH, Stead LF. Nursing interventions for smoking cessation (Review). Cochrane Database of Systematic Reviews. 2004; Issue 1.

12. O'Donovan G. Smoking prevalence among qualified nurses in the Republic of Ireland and their role in smoking cessation. International Nursing Review. 2009;56:230-6.

13. Green MA. Tobacco use among individuals with mental illness: nurses' knowledge, confidence, attitudes and practice [Master of Nursing]. Winnipeg: Faculty of Nursing, University of Manitoba; 2010.

14. Ingersoll GI. Evidence-based nursing: what it is and what it isn't. Nursing Outlook. 2000;48:151-2.

15. Driever MJ. Are evidenced-based practice and best practice the same? Western Journal of Nursing Research. 2002;24(5):591-7.

16. Galvão CM, Sawada NO, Mendes IAC. A busca das melhores evidências. Revista Escola de Enfermagem USP. 2003;37(4):43-50.

17. Kirkevold M. Integrative nursing research: an important strategy to further the development of nursing science and nursing practice. Journal of Advanced Nursing. 1997;25:977-84.

18. Whittemore $R$, Knafl $K$. The integrative review: updated methodology. Journal of Advanced Nursing. 2005;52(5):546-53.

19. Mendes KDS, Silveira RCCP, Galvão CM. Revisão integrativa: método de pesquisa para a incorporação de evidências na saúde e na enfermagem. Texto \& Contexto Enfermagem. 2008;17(4):758-64.

20. Ursi ES, Galvão CM. Prevenção de lesões de pele no perioperatório: revisão integrativa da literatura. Revista Latino-americana de Enfermagem. 2006;14(1):124-31.

21. Melnyk BM, Fineout-Overholt E. Evidence based practice in nursing \& healthcare: a guide to best practice. Philadelphia: Lippincott Williams \& Wilkins; 2005.

22. Shinozaki Y, Nakao M, Takeuchi T, et al. Smoking rates among schizophrenia patients in Japan. Psychiatry Research. 2011;186:165-9.

23. Johnson JL, Ratner PA, Malchy LA, et al. Gender-specific profiles of tobacco use among non-institutionalized people with serious mental illness. BMC Psychiatry.2010;10(101). 
24. Ma X, Li C, Meng $\mathrm{H}$, et al. Premorbid tobacco smoking is associated with later age at onset in schizophrenia. Psychiatry Research. 2010;178:461-6.

25. Wang CY, Xiang YT, Weng YZ, et al. Cigarette smoking in patients with schizophrenia in China: prospective, multicentre study. Australian and New Zealand Journal of Psychiatry. 2010;44:456-62.

26. Kotov R, Guey LT, Bromet EJ, et al. Smoking in schizophrenia: diagnostic specificity, symptoms correlates and illness severity. Schizophrenia Bulletin. 2010.36(1):173-81.

27. Galazyn M, Steinberg ML, Gandhi KK, et al. Reasons for smoking among individuals with schizophrenia. Schizophrenia Research. 2010;122:268-9.

28. Zhang $X Y$, Zhang $R L$, Pan $M$, et al. Sex difference in the prevalence of smoking in Chinese schizophrenia. Journal of Psychiatric Research.2010;44:986-8.

29. Zhang $X Y$, et al. Smoking initiation and schizophrenia: a replication study in a Chinese Han population. Schizophrenia Research. 2010;119:110-14.

30. Yip SW, Sacco KA, George TP, et al. Risk/reward decision-making in schizophrenia: a preliminary examination of the influence of tobacco smoking and relationship to Wisconsin Card Sorting Task Performance. Schizophrenia Research. 2009;110(1-3):156, 2009.

31. Diaz FJ, James D, Botts $S$, et al. Tobacco smoking behaviors in bipolar disorder: a comparison of the general population, schizophrenia, and major depression. Bipolar Disorders. 2009;11:154-65.

32. Subramaniam M, Cheok C, Lee IM, et al. Nicotine dependence and psychiatric disorders among young males in Singapore. Nicotine \& Tobacco Research. 2009;11(9):1107-13.

33. Chapman S, Ragg M, McGeechan K. Citation bias in reported smoking prevalence in people with schizophrenia. Australian and New Zealand Journal of Psychiatry. 2009;43:27782.

34. Diehl A, Reinhard I, Schmitt A, et al. Does the degree of smoking effect the severity of tardive dyskinesia? A longitudinal clinical trial. European Psychiatry. 2009;24:33-40.

35. Barr AM, Procyshin RM, Hui P, et al. Self-reported motivation to smoke in schizophrenia is related to antipsychotic drug treatment. Schizophrenia Research. 2008;100:252-60.

36. Diaz FJ, Velásquez DM, Susce MT, et al. The association between schizophrenia and smoking: unexplained by either the illness or the prodromal period. Schizophrenia Research. 2008;104:214-9.

37. Fawzi MH, Fawzi MM, Khedr $\mathrm{HH}$, et al. Tobacco smoking in Egyptian schizophrenia patients with and without obsessive compulsive symptoms. Schizophrenia Research. 2007;95:236-46.

38. Tang YL, George TP, Mao PX, et al. Cigarette smoking in Chinese male inpatients with schizophrenia: a cross-sectional analysis. Journal of Psychiatric Research. 2007;41:43-8.

39. Baker A, Richmond R, Haile M, et al. Characteristics of smokers with psychotic disorder and implications for smoking interventions. Psychiatry Research. 2007;150:141-52.

40. Levander S, Eberhard J, Lindstrom E. Nicotine use and its correlates in patients with psychosis. Acta Psychiatrica Scandinavica. 2007;116(435 Suppl):27-32.

41. Gurpegui M, Martínez-Ortega JM, Jurado D, et al. Subjective effects and the main reason for smoking in outpatients with schizophrenia: a case-control study. Comprehensive Psychiatry. 2007;48:186-91.

42. Campo-Arias A, Diaz-Martínez LA, Rueda-James GE, et al. Smoking is associated with schizophrenia, but not with mood disorders, within a population with low smoking rates: a matched case-control study in Bucaramanga, Colombia. Schizophrenia Research. 2006;83:269-76.

43. Dome P, Teleki Z, Gonda X, et al. Relationship between obsessive-compulsive symptoms and smoking habits amongst schizophrenic patients. Psychiatry Research. 2006;144:227-31.

44. Morris CD, Giese AA, Turnbull JJ, et al. Predictors of tobacco use among persons with mental illnesses in a Statewide population. Psychiatric Services. 2006;57(7):1035-38. 
45. Salokangas RKR, Honkonen T, Stengard E, et al. Cigarette smoking in long term schizophrenia. European Psychiatry. 2006;21:219-23.

46.Barnes M, Lawford BR, Burton SC, et al. Smoking and schizophrenia: is symptom profile related to smoking and which antipsychotic medication is of benefit in reducing cigarette use? Australian and New Zealand Journal of Psychiatry. 2006;40:575-80.

47. Williams JM, Ziedonis DM, Abanyie F, et al. Increased nicotine and cotinina levels in smokers with schizophrenia and shizoaffective disorder is not a metabolic effect. Schizophrenia Research. 2005;79:323-35.

48. Gurpegui M, Martínez-Ortega JM, Aguilar C, et al. Smoking initiation and schizophrenia: a replication study in a Spanish sample. Schizophrenia Research. 2005;76:113-18.

49. TIDEY JW, Rohsenow DJ, Kaplan GB, et al. Cigarette smoking topography in smokers with schizophrenia and matched non-psychiatric controls. Drug and Alcohol Dependence. 2005;80:259-65.

50. Esterberg ML, Compton MT. Smoking behavior in persons with a schizophrenia-spectrum disorder: a qualitative investigations of the transtheoretical model. Social Science \& Medicine. 2005;61:293-303.

51. Riala K, Hakko H, Isohanni M, et al. Poor premorbid school performance is associated with later cigarette smoking among schizophrenia patients? Psychiatry Research. 2005;137:137-41.

52. Riala K, Hakko $\mathrm{H}$, Isohanni $\mathrm{M}$, et al. Is initiation of smoking associated with the prodromal phase of schizophrenia? Rev Psychiatr Neuroscience. 2005;30(1):26-32.

53. Weiser M., Reichenberg A, Grotto I, et al. Higher rates of cigarette smoking in male adolescents before the onset of schizophrenia: a historical-prospective cohort study. American Journal of Psychiatry. 2004;161:1219-23.

54. Etter M, Mohr S, Garin C, et al. Stages of change in smokers with schizophrenia or schizoaffective disorder and in the general population. Schizophrenia Bulletin. 2004;30(2):459-68.

55. Dervaux A, Baylé FJ, Laqueille $X$, et al. Nicotine use in schizophrenia and desinhibition. Psychiatry Research. 2004;128:229-34.

56. Uçok A, Polat A, Bozkurt $O$, et al. Cigarette smoking among patients with schizophrenia and bipolar disorders. Psychiatry and Clinical Neuroscience. 2004;58:434-7.

57. Spring B, Pingitore R, McChargue DE. Reward value of cigarette smoking for comparably heavy smoking schizophrenic, depressed, and nonpatient smoker. American Journal of Psychiatry. 2003;160:316-22.

58. Zammit S, Allebeck $\mathrm{P}$, Dalman $\mathrm{C}$, et al. Investigating the association between cigarette smoking and schizophrenia in a cohort study. American Journal of Psychiatry. 2003;160:2216-21.

59. Uzun O, Cansever A, Basoglu C, et al. Smoking and substance abuse in outpatients with schizophrenia: a 2-year follow up study in Turkey. Drug and Alcohol Dependence. 2003;70:187-92.

60. Mori T, Sasaki T, Iwanami A, et al. Smoking habits in Japanese patients with schizophrenia. Psychiatry Research. 2003;120:207-9.

61. De Leon J, Tracy J, McCann E, et al. Schizophrenia and tobacco smoking: a replication study in another US psychiatric hospital. Schizophrenia Research. 2002;56:55-65.

62. Srinivasan TN, Tara R. Smoking in schizophrenia - all is not biological. Schizophrenia Research. 2002;56:67-74.

63. De Leon J, Diaz FJ, Rogers T, et al. Initiation of daily smoking and nicotine dependence in schizophrenia and mood disorders. Schizophrenia Research. 2002;56:47-54.

64. Patkar AA, Gopalakrishnan R, Lundy A, et al. Relationship between tobacco smoking and positive and negative symptoms in schizophrenia. The Journal of Nervous and Mental Disease. 2002;190(9):604-10. 
65. Beratis S, Katrivanou A, Gourzis P. Factors affecting smoking in schizophrenia. Comprehensive Psychiatry. 2001;42(5):393-402.

66. Winterer G. Why do patients with schizophrenia smoke? Current Opinion in Psychiatry. 2010;23:112-9.

67. Prochaska JJ, Hall SM, Bero LA. Tobacco use among individuals with schizophrenia: what role has the Tobacco Industry Played? Schizophrenia Bulletin. 2008;34(3):555-67.

68. Keltner NL, Grant JS. Smoke, smoke, smoke that cigarette. Perspectives in Psychiatric Care. 2006;42(4):256-61.

69. Calheiros PRV, Oliveira MS, Andretta I. Comorbidades psiquiátricas no tabagismo. Aletheia. 2006;23:65-74.

70. De Leon J, Diaz FJ. A meta-analysis of worldwide studies demonstrates an association between schizophrenia and tobacco smoking behaviors. Schizophrenia Research. 2005;76:135-57.

71. Steinberg ML, Williams JM, Ziedonis DM. Financial implications of cigarette smoking among individuals with schizophrenia. Tobacco Control.2004;13:206-8.

72. Rondina RC, Gorayeb R, Botelho C. Relação entre tabagismo e transtornos psiquiátricos. Revista de Psiquiatria Clínica. 2003;30(6):221-8.

73. Breen MJ. The incidence of lung cancer among schizophrenic males versus nonschizophrenic males [Dissertation]. United States: Education United States International University; 1981.

74. Parfitt DN. Lung câncer and schizophrenia. British Journal of Psychiatry. 1981;138:17980.

75. Lichtermann D, Ekelund J, Pukkala E, et al. Incidence of cancer among persons with schizophrenia and their relatives. Archives of General Psychiatry. 2001;58:573-8.

76. Sadock BJ, Sadock VA. Kaplan \& Sadock compêndio de psiquiatria: ciência do comportamento e psiquiatria clínica. 9 ed. Porto Alegre: Artmed; 2007.

77. Lyon ER. A review of the effects of nicotine on schizophrenia and antiphychotic medications. Psychiatric Services. 1999;50(10):1346-50.

78. Dalack GW, Healy D, Meador-Woodruff JH. Nicotine dependence in schizophrenia: clinical phenomena and laboratory findings. American Journal of Psychiatry. 1998;155(11):1490-1501.

79. Chen XS, Li CB, Smith RC, et al. Differential sensory gating functions between smokers and non-smokers among drug-naïve first episode schizophrenic patients. Psychiatry Research. 2011. No prelo.

80. Solway E. Windows of opportunity for culture change around tobacco use in mental health settings. Journal of the American Psychiatric Nurses Association. 2009;15(1):41-9.

81. Ziedonis DM, George TP. Schizophrenia and nicotine use: report of pilot smoking cessation program and review of neurobiological and clinical issues. Schizophrenia Bulletin. 1997;23:247-54.

82. Sarna L, Wewers ML, Brown JK, et al. Barriers to tobacco cessation in clinical practice: report from a national survey of oncology nurse. Nursing Outlook. 2001;49(4):166-72.

83. Gomm M, Lincoln $P$, Egeland $P$, et al. Helping hospitalised clients quit smoking: a study of rural nursing practice and barriers. Australian Journal of Rural Health. 2002;10(1):26-32.

84. Essenmacher C, Karvonen-Gutierrez C, Lynch-Sauer J, et al. Staff's attitudes toward the delivery of tobacco cessation services in a primarily psychiatric veterans affairs hospital. Archives of Psychiatric Nursing. 2008;23(3):231-42.

85.Robson D, Gray R. Serious mental illness and physical health problems: a discussion paper. International Journal of Nursing Studies. 2007;44:457-66. 
